\title{
COMPARATIVO ENTRE LÂMPADAS FLUORESCENTES E LÂMPADAS DE LED SOB A VISÃO DO SUPERDIMENSIONAMENTO EM SUA SUBSTITUIÇÃO
}

Tárcis Reis Pinto

Graduado em Engenharia Elétrica pelo Centro Universitário Augusto Motta - RJ - Brasil

tarcis.reis.trp@gmail.com

\section{Geraldo Motta Azevedo Júnior}

Doutor em Engenharia Elétrica pela COPPE/UFRJ - RJ - Brasil

Professor do Centro Universitário Augusto Motta - UNISUAM - RJ - Brasil

geraldomotta@unisuam.edu.br

\section{RESUMO}

O presente artigo aborda como o avanço da tecnologia e incentivos para a redução de consumo de energia elétrica são questões que têm crescido no mercado nacional e internacional. Atualmente as lâmpadas fluorescentes convencionais têm sido substituídas por lâmpadas de LED visando à redução de consumo de energia elétrica apresentando uma melhor eficiência. Este trabalho apresenta um estudo comparativo entre as duas tecnologias de modo a demonstrar a eficiência luminosa entre as tecnologias para que durante o processo de retrofit não ocorra superdimensionamento no quesito de luminosidade necessária para o ambiente em relação às normas vigentes que determinam os valores de luminosidade para o desenvolvimento de atividades. O trabalho foi realizado com o auxílio de um software de cálculos luminotécnicos chamado DIALux, em que é possível utilizar dados fornecidos diretamente de diferentes fabricantes de lâmpadas e luminárias. $O$ estudo apresenta resultados para diferentes ambientes onde foi possível demonstrar a superioridade das lâmpadas de LED em comparação com as lâmpadas fluorescentes convencionais, representando assim a possível redução dos quantitativos permanecendo com a eficiência luminosa de acordo com as normas vigentes. O estudo conclui que de acordo com as atividades realizadas torna-se viável a redução do quantitativo de lâmpadas ou luminárias, permitindo assim a redução de potência instalada e, consequentemente, o consumo de energia elétrica, com a vantagem adicional da redução de manutenção nos sistemas de iluminação. 
Palavras-chave: Lâmpadas Fluorescentes, Lâmpadas de LED, Superdimensionamento.

\title{
COMPARATIVE BETWEEN FLUORESCENT LAMPS AND LED LAMPS UNDER THE VIEW OF SUPERDIMENSIONING IN ITS SUBSTITUTION
}

\begin{abstract}
The present article addresses how the advancement of technology and incentives for the reduction of electric energy consumption are issues that have grown in the national and international market. Nowadays conventional fluorescent lamps have been replaced by LED lamps aiming at reducing energy consumption by presenting better efficiency. This work presents a comparative study between the two technologies in order to demonstrate the luminous efficiency between the technologies so that during the retrofit process there is no oversizing in the area of luminosity necessary for the environment in relation to the current norms that determine the luminosity values for the development of activities. The work was carried out with the aid of a lighting calculator software called DIALux, in which it is possible to use data supplied directly from different manufacturers of lamps and luminaires. The study presents results for different environments where it was possible to demonstrate the superiority of LED lamps compared to conventional fluorescent lamps, thus representing the possible reduction of the quantitative ones remaining with the luminous efficiency according to the current norms. The study concludes that according to the activities performed it is possible to reduce the quantity of lamps or luminaires, thus allowing the reduction of installed power and, consequently, the consumption of electric energy, with the additional advantage of the reduction of maintenance in the systems lighting.
\end{abstract}

Keywords: Fluorescent Lamps, LED Lamps, Over-dimensioning.

\section{INTRODUÇÃO}

Devido ao avanço tecnológico e forte incentivo pela redução de consumo de energia elétrica, as grandes indústrias têm desenvolvido lâmpadas capazes de 
proporcionar as mesmas características no aspecto de emissão de luz, porém consumindo-se menos energia para realizar este trabalho.

Esta tecnologia desenvolvida se deu graças ao advento dos componentes eletrônicos denominados LEDs (Light Emitting Diodes) para a substituição dos gases presentes nas lâmpadas fluorescentes convencionais.

Conforme a tecnologia LED avança, tem-se observado um grande problema durante o chamado retrofit (Processo de modernização de instalações ou equipamentos); na execução deste processo, frequentemente não são reavaliadas as questões técnicas presentes para a aquisição deste novo produto ocasionando um superdimensionamento da quantidade de luz necessária de acordo com a atividade a ser desenvolvida no local.

Realizando um estudo antes do processo de modernização dos sistemas de iluminação, é possível muitas das vezes reduzir o número de lâmpadas/luminárias mantendo a eficiência luminosa necessária para a atividade desenvolvida, evitando assim o superdimensionamento de lâmpadas de LED em ambientes internos e externos durante a modernização de sistemas de iluminação e impedindo o excesso de luminosidade e consumo excessivo de energia elétrica.

O objetivo geral deste trabalho é apresentar um estudo comparativo entre duas tecnologias de lâmpadas existentes visando reduzir o superdimensionamento de luminosidade na modernização de sistemas de iluminação existentes. Inicialmente são discutidas as diferenças entre as duas tecnologias estudadas (Lâmpadas Fluorescentes $x$ Lâmpadas LED). Em seguida, são apresentadas simulações evidenciando as diferenças na utilização dos diferentes tipos de lâmpadas em algumas áreas de utilização determinadas. A partir da análise destes resultados simulados verifica-se a possibilidade de redução do número de lâmpadas mantendo a mesma eficiência luminosa determinada de acordo com a utilização de cada ambiente.

Em seu trabalho de conclusão de curso (FERREIRA, 2014) aborda a comparação entre lâmpadas fluorescentes tubulares T8 e lâmpadas tubulares de LED com o intuito de verificar o desempenho luminoso e viabilidade econômica no processo de substituição das lâmpadas fluorescentes convencionais.

Valquíria Aparecida Alcantara Lima realizou em seu trabalho de conclusão de curso 
(LIMA, 2013) um estudo apresentando os benefícios na substituição de lâmpadas convencionais por lâmpadas de LED de alta potência, através de parâmetros fotométricos, consumo de energia elétrica e análise econômica visando também à redução do consumo energético.

Um artigo (PADILHA; JUNG; RODRIGUES, 2015) publicado na VIII Mostra Nacional de Iniciação Cientifica e Tecnológica Interdisciplinar (MICTI) em 2015 relatou um estudo de caso referente ao Instituto Federal Catarinense - campus Luzerna, uma análise comparativa entre lâmpadas fluorescentes tubulares e lâmpadas tubulares de LED verificando o custo de implementação, manutenção e descarte de resíduos sólidos.

Conforme o avanço tecnológico e fim da comercialização de lâmpadas incandescentes no Brasil em 30 de junho de 2016 (PORTAL BRASIL, 2016), o incentivo à utilização de lâmpadas mais econômicas tem crescido bastante, aparecendo no mercado nacional diversos fabricantes com diferentes características.

Para a utilização de lâmpadas LED é necessário observar alguns critérios importantes durante a escolha do modelo de lâmpada para se evitar três principais fatores:

1. Excesso de luminosidade num ambiente de acordo com sua utilização;

2. Desconforto visual para as pessoas que utilizem o ambiente;

3. Consumo de energia desnecessário;

Os dois primeiros fatores apresentados são de grande valia, pois estes podem causar problemas de visão em pessoas expostas a estas condições durante longos períodos de tempo. Um dos problemas fisiológicos que podem ocorrer devido ao excesso de luminosidade num ambiente é a síndrome da disfunção lacrimal, conhecida como síndrome do olho seco (IORJ, 2013), uma disfunção lacrimal que é:

"um grupo de diferentes doenças e condições que resultam da lubrificação inadequadas dos olhos com diminuição da produção da lágrima ou deficiência em alguns de seus componentes, ou seja, pouca quantidade e/ou má qualidade da lágrima". (IORJ, 2013).

O superdimensionamento de lâmpadas de LED em ambientes internos e externos durante a modernização de sistemas de iluminação ocasiona excesso de luminosidade o 
que pode causar danos à saúde visual das pessoas expostas a estes ambientes e, ainda, provocar um consumo de energia elétrica desnecessário, tendo em vista a possibilidade da redução do número de lâmpadas para evitar tal superdimensionamento de luminosidade.

\section{DESENVOLVIMENTO}

Para melhor entendimento deste trabalho se faz necessário apresentar alguns conceitos básicos que servem de base para a compreensão do estudo.

\subsection{Luminotécnica}

Luminotécnica é denominado o estudo da implementação e utilização de iluminação artificial em ambientes internos e externos.

A Luminotécnica é bastante antiga, inclusive anterior à utilização de energia elétrica para a geração de iluminação artificial, pois anteriormente as fontes de iluminação artificial eram oriundas de lamparinas a óleo ou gás e até mesmo tochas de fogo.

\section{$2.2 \mathrm{Luz}$}

A luz pode ser definida como sendo formas de onda de radiação eletromagnéticas que possuem espectros visíveis e não visíveis ao olho humano. As ondas de radiação eletromagnética não necessitam de um meio para se propagarem no espaço, podendo ser propagadas inclusive no vácuo. "A luz visível refere-se ao conjunto de ondas capaz de provocar sensação num observador normal." (SALVETTI, 2008, p. 9).

Pode-se afirmar que a luz visível é uma parcela das formas de onda do espectro eletromagnético. O olho humano é capaz de perceber apenas uma faixa do comprimento de ondas eletromagnéticas que estão compreendidas entre 380 e 780 nm (nanômetros). A figura 1 apresenta os comprimentos de onda desde raios gama até as ondas de rádio. 
Figura 1 - Espectro visível da Luz

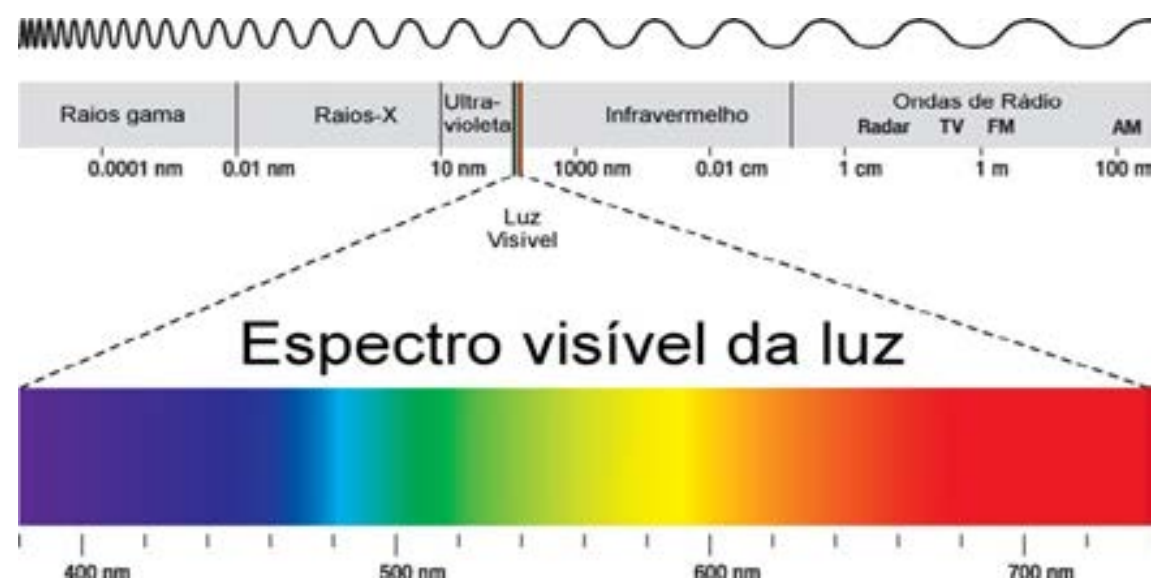

Fonte: (ELEUTÉRIO, 2012)

\subsection{Fluxo luminoso}

Fluxo luminoso é toda a radiação emitida por uma fonte luminosa dentro dos limites de comprimento de onda visível (380 a $780 \mathrm{~nm}$ ) em todas as direções.

O fluxo luminoso (figura 2) também pode ser definido pela quantidade de luz emitida por uma fonte luminosa em sua tensão nominal de funcionamento (LUMICENTER, 2017). O símbolo do fluxo luminoso é $\varphi$ e a unidade lúmen (Im).

Figura 2 - Fluxo Luminoso

Fonte: (OSRAM, 2007) 
2.4 lluminância ou iluminamento

Iluminância ou iluminamento representado pela figura 3 é a grandeza que mede o fluxo luminoso emitido por uma fonte de luz que incide sobre uma superfície situada a certa distância desta fonte, ou seja, mede a quantidade de luz que chega a um determinado ponto.

Esta relação é dada entre a intensidade luminosa e o quadrado da distância $\left(I / d^{2}\right)$, ou ainda, entre o fluxo luminoso e a área da superfície. A iluminância pode ser medida através de um luxímetro, porém não pode ser vista, o que é possível visualizar são as diferenças na reflexão da luz.

Figura 3 - Iluminância

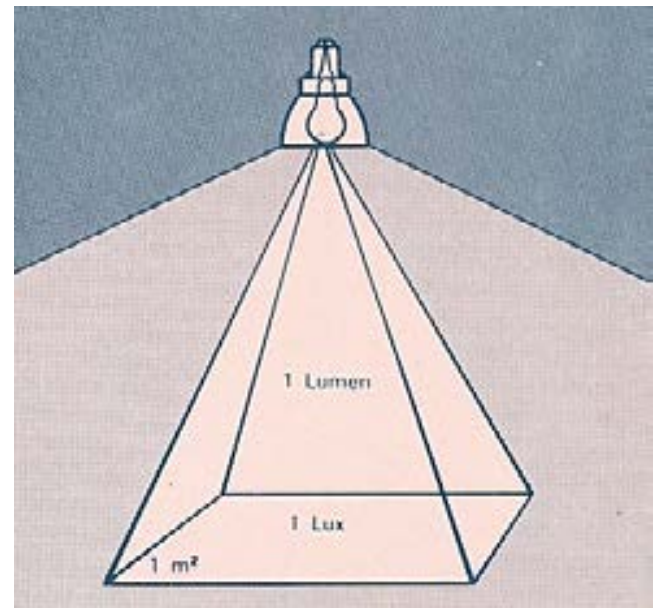

Fonte: (FAU, 2013)

2.5 Eficiência luminosa ou rendimento luminoso

A relação entre fluxo luminoso e potência consumida é denominada Eficiência Luminosa ou Rendimento Luminoso. Esta relação permite comparar diferentes tipos de lâmpadas observando quantos lúmens são fornecidos por watt consumido (OSRAM, 2007). A figura 4 apresenta a eficiência luminosa fornecida de acordo com o tipo de lâmpada. 
Figura 4 - Eficiência Luminosa

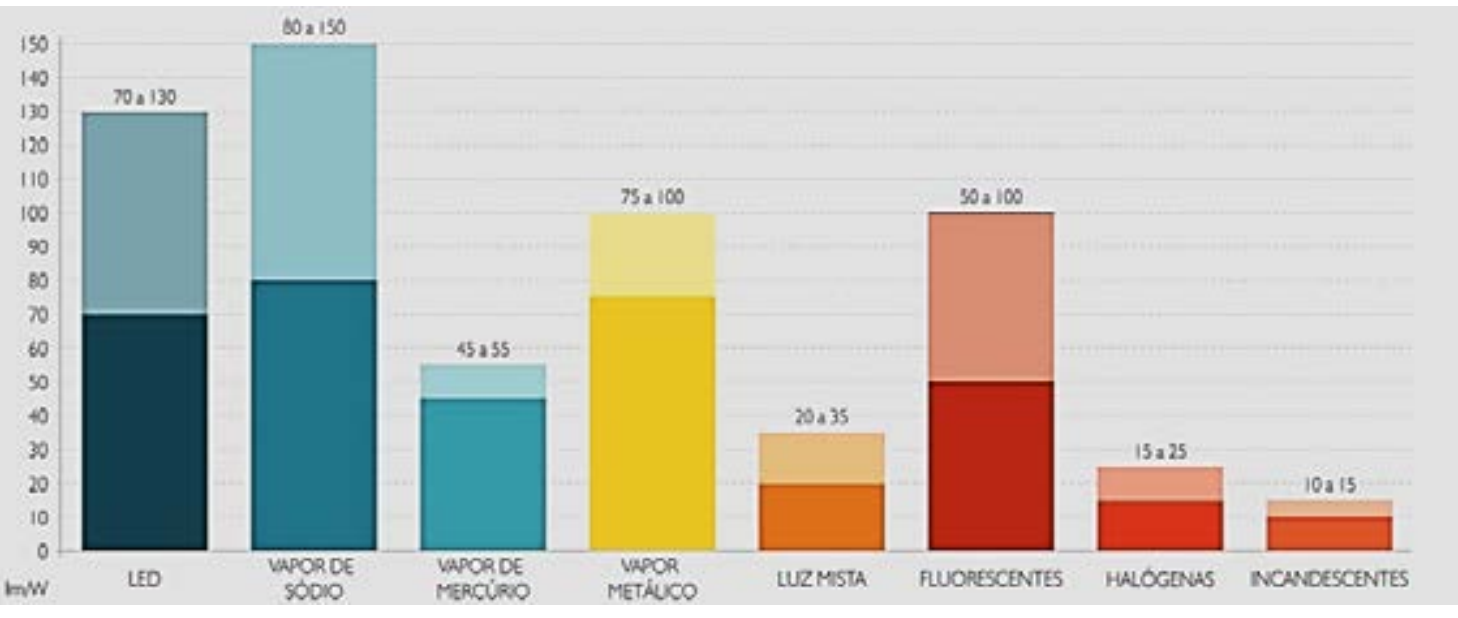

Fonte: Site EMPALUX

\subsection{Normas e Regulamentações}

Os sistemas de iluminação, tanto internos como externos, são regidos por normas e regulamentações nacionais (NBR / ABNT) ou internacionais (ISO / IEC). Estas normas fornecem as diretrizes e especificações mínimas para a elaboração de projetos de iluminação de acordo com o tipo de atividade a ser desenvolvida.

No Brasil atualmente é utilizada a NBR 8995:2013 - Iluminação de Ambientes de Trabalho, que substituiu a antiga NBR 5413:1992 - lluminância de Interiores em 21 de março de 2013. Entretanto, a NBR 5413:1992 ainda é utilizada para a determinação de alguns parâmetros que permaneceram durante a atualização para a NBR 8995:2013.

\subsection{Simulações e resultados}

Serão apresentadas as simulações comparativas entre as duas tecnologias de lâmpadas predominantes no mercado, lâmpadas fluorescentes e lâmpadas de LED.

- Ambiente Interno: Ambiente sem iluminação natural no qual se necessita atender o fluxo luminoso estabelecido pela NBR 8995:2013 de acordo com a atividade a ser executada no ambiente. Foi considerado o ambiente de Sala de Aula como ambiente de referência; 
- Ambiente Externo: Ambiente com iluminação natural presente onde se necessita realizar um sistema de iluminação artificial para melhor atender a atividade a ser executada segundo a NBR 8995:2013 e NBR 5101:2012. Foi considerado o ambiente de Estacionamento Descoberto do Bangu Shopping como ambiente de referência;

2.8 Simulação de Ambiente Interno - Salas de Aula

Para este ambiente foram realizadas três simulações para um mesmo modelo padrão de Sala de Aula com dimensões de 7 metros de comprimento por 10 metros de profundidade, pé direito de 3 metros e superfície de plano de trabalho de 0,75 metro.

De acordo com a NBR 8995:2013 para atividades de Salas de Educação a iluminância mantida () deve ser de 500 lux.

Para a primeira simulação foi utilizada a lâmpada fluorescente tubular modelo Lumilux Deluxe T8 - L30W/930 do fabricante Osram. O layout utilizado para a simulação com lâmpadas fluorescentes para atender a NBR 8995:2013 foi o apresentado na figura 5, contendo 12 Luminárias com duas lâmpadas cada 
Figura 5 - Layout de Luminárias com Lâmpadas Fluorescentes - Sala de Aula

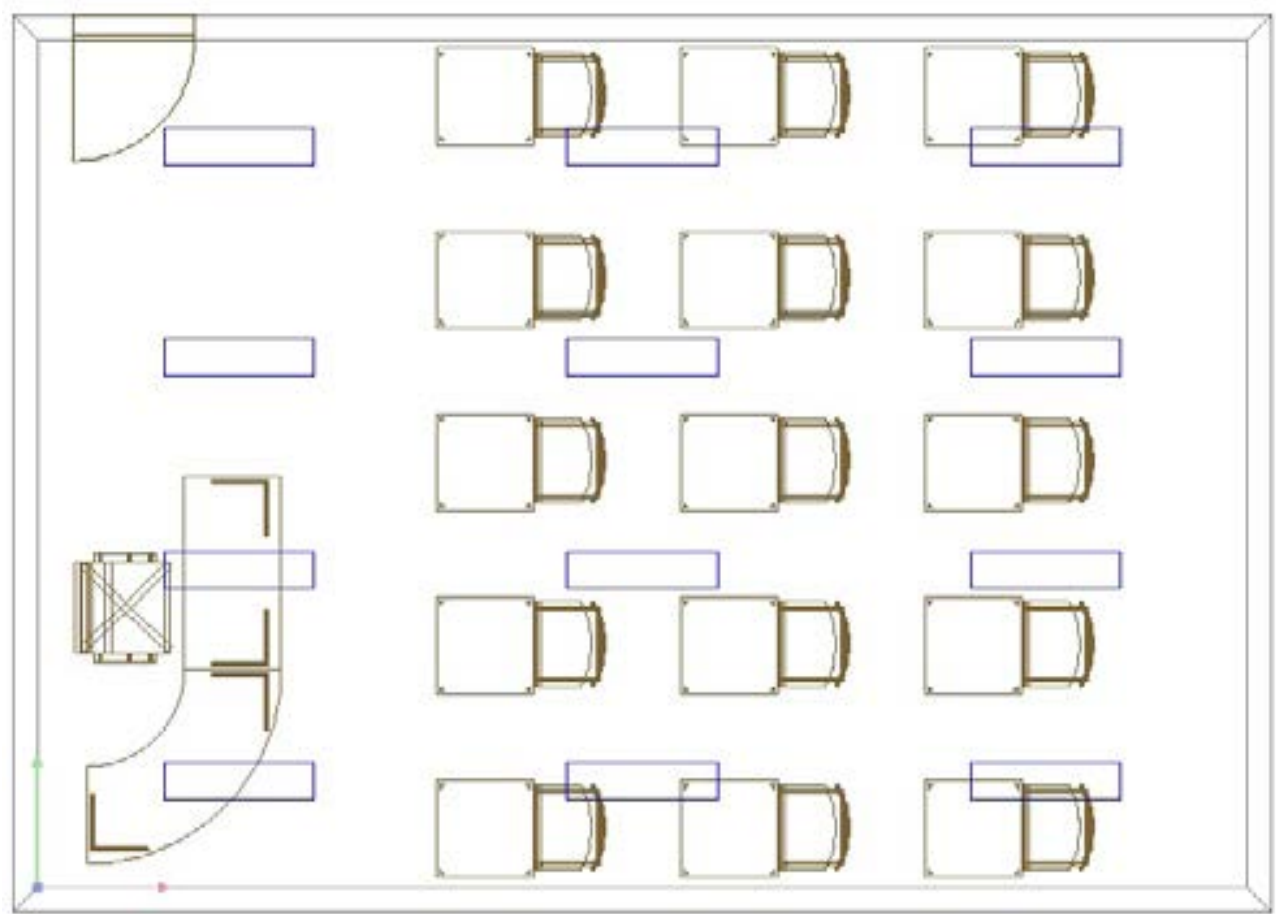

Fonte: (Autor, 2017)

De acordo com o layout apresentado pela figura 5, foram obtidos os seguintes resultados de iluminância mantida ().

Tabela 1 - Resultado lluminância Mantida () Lâmpada Fluorescente - Sala de Aula

\begin{tabular}{|c|c|c|c|c|}
\hline$E m($ Lux) & $E m i n(L u x)$ & $E m a x(L u x)$ & $E m i n / E m$ & $E m i n / E m a x$ \\
\hline 580 & 409 & 709 & 0,704 & 0,576 \\
\hline
\end{tabular}

Fonte: (Autor, 2017)

Para a segunda simulação manteve-se o layout de luminárias (figura 5). No entanto, foram substituídas as lâmpadas fluorescentes tubulares por lâmpadas tubulares de LED e considerada a utilização da lâmpada tubular de LED modelo ST8-HA4 22W/830 do fabricante Osram. 
Utilizando a lâmpada tubular de LED, foram obtidos os seguintes resultados de iluminância mantida ().

Tabela 2 - Resultado Iluminância Mantida () Lâmpada LED - Sala de Aula

\begin{tabular}{|c|c|c|c|c|}
\hline$E m(L u x)$ & $E \min ($ Lux $)$ & $E \max ($ Lux $)$ & $E \min / E m$ & $E \min /$ Emax \\
\hline 675 & 542 & 748 & 0,803 & 0,725 \\
\hline \multicolumn{5}{|c}{ Fonte: (Autor, 2017) } \\
\hline
\end{tabular}

Para a terceira simulação, foi realizado um novo layout de luminárias (figura 6) reduzindo de 12 para 9 pontos de luz no ambiente proposto e utilizando a mesma lâmpada tubular de LED modelo ST8-HA4 22W/830 do fabricante Osram, utilizada anteriormente.

Figura 6 - Novo Layout de Luminárias com Lâmpadas de LED - Sala de Aula

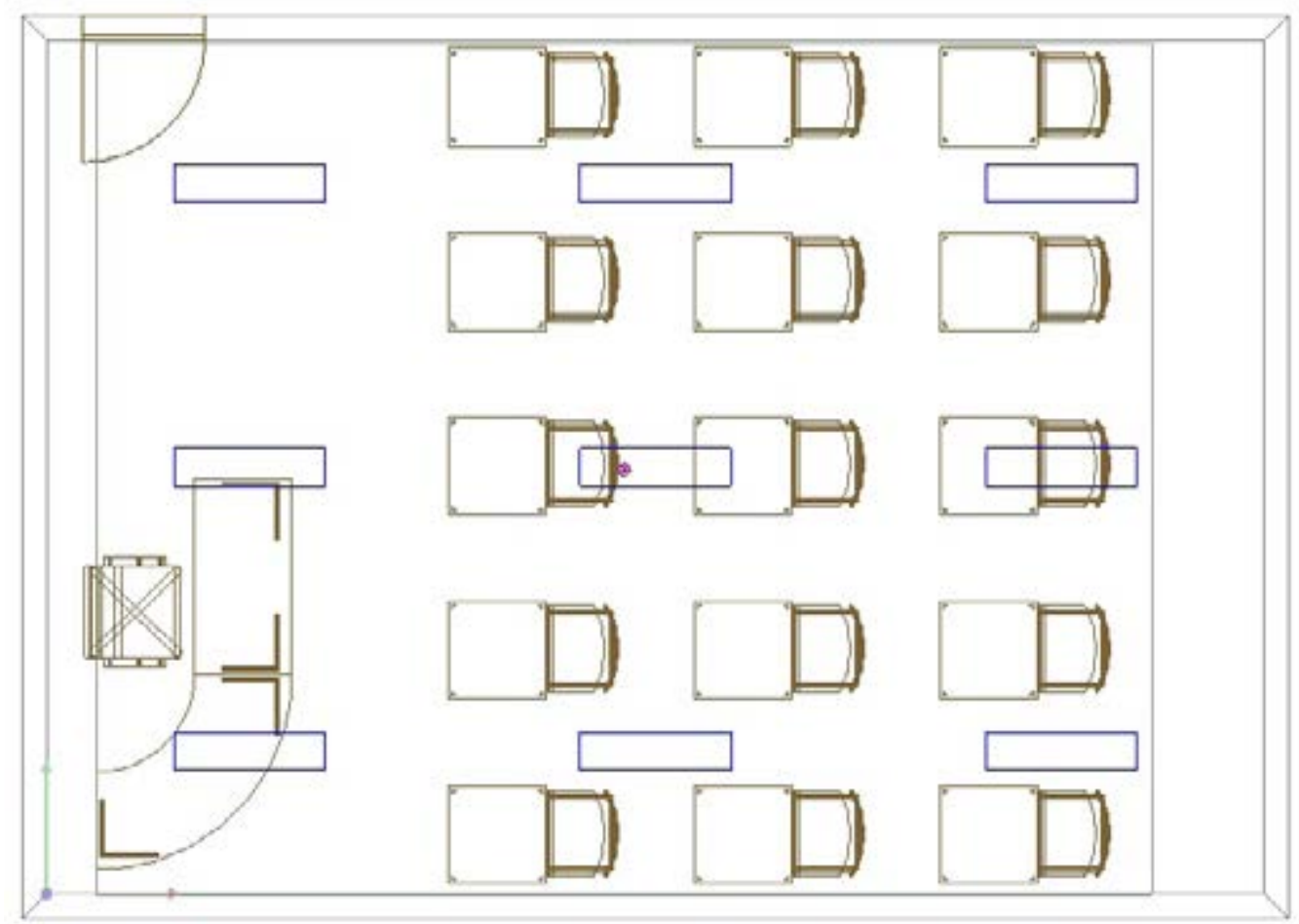

Fonte: (Autor, 2017) 
De acordo com o layout apresentado pela figura 6, foram obtidos os seguintes resultados de iluminância mantida ().

Tabela 3 - Resultado Iluminância Lâmpada LED com menos pontos - Sala de Aula

\begin{tabular}{|c|c|c|c|c|}
\hline$E m(L u x)$ & $E m i n(L u x)$ & $E \max ($ Lux $)$ & $E \min / \mathrm{Em}$ & $\mathrm{Emin} / \mathrm{Emax}$ \\
\hline 523 & 417 & 579 & 0,798 & 0,721 \\
\hline
\end{tabular}

Fonte: (Autor, 2017)

Verifica-se que após a redução dos pontos de luz no ambiente de Sala de Aula utilizando lâmpadas de LED, a iluminância mantida () permaneceu dentro do padrão requerido pela NBR 8995/2013, que determina o valor de 500 Lux para a atividade exercida em Salas de Aula.

Figura 7 - Comparativos Resultados - Ambiente Interno Sala de Aula

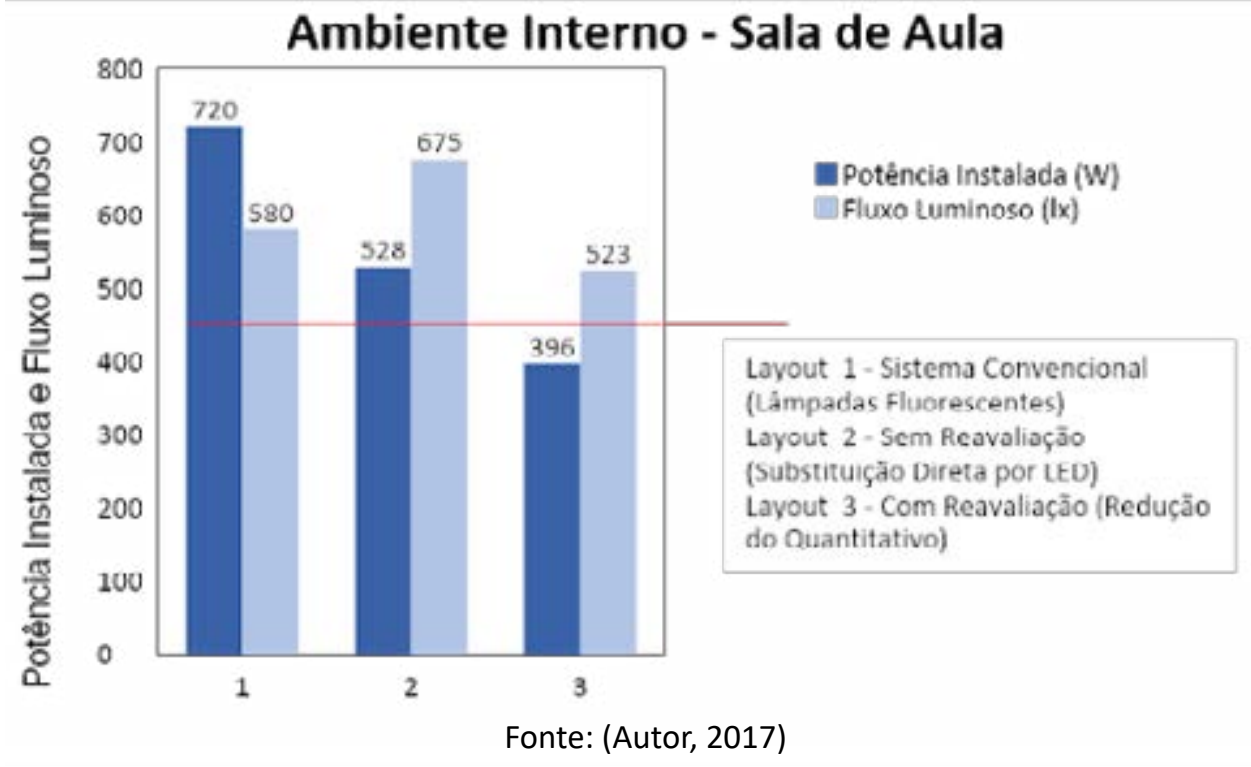

Conclui-se que para este ambiente de Sala de Aula foi possível a redução de luminárias no ambiente mantendo a iluminância mantida () solicitada pela NBR 8995/2013. Isto representa também a redução de potência instalada inicialmente no valor de $720 \mathrm{~W}$ para um valor final de $396 \mathrm{~W}$, isto é, em torno de $45 \%$ de redução de potência instalada ainda fornecendo uma iluminância mantida () de 523 Lux para a atividade a ser exercida 
atendendo a NBR 8995/2013, conforme apresentado na figura 7.

2.9 Simulação de Ambiente Externo - Estacionamento Descoberto

Para a simulação deste ambiente, foi considerado um ambiente real para que seja possível demonstrar a eficácia do estudo realizado. O ambiente proposto para a simulação de Estacionamento Descoberto foi um trecho do estacionamento do Shopping Bangu conforme é mostrado na figura 8.

Figura 8 - Trecho do Estacionamento Bangu Shopping

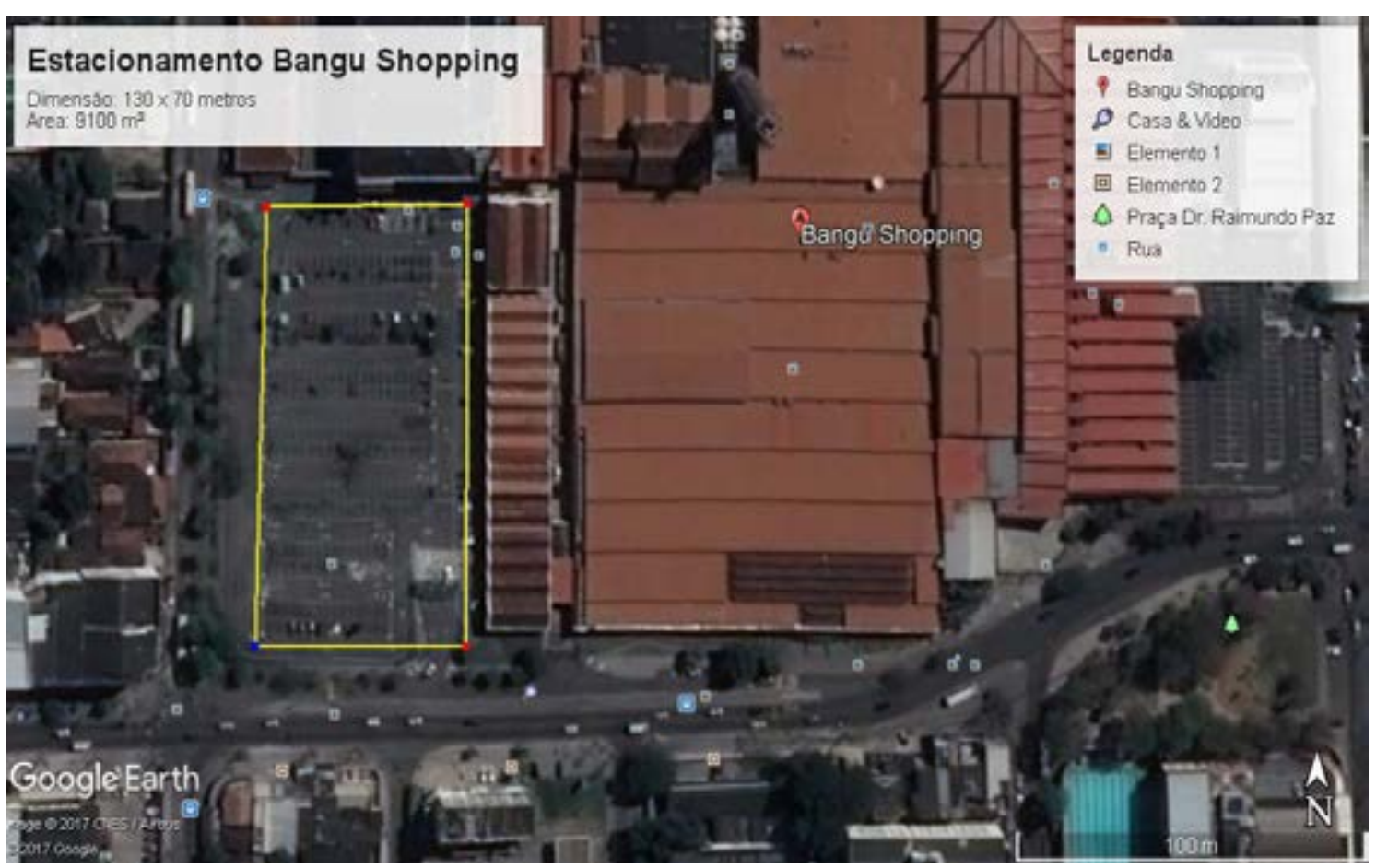

Fonte: (Adaptado de Google Earth, 2017)

Para o trecho considerado as simulações foram realizadas para o mesmo modelo padrão (figura 9) de Estacionamento Descoberto com dimensões de 35 metros de comprimento por 65 metros de profundidade, altura de montagem de 15 metros e superfície de plano de trabalho 0,75 metro.

De acordo com a NBR 8995/2013 para atividades de Estacionamentos Internos a 
iluminância mantida () deve ser de 75 Lux. A NBR 8995/2013 também recomenda por questões de segurança uma iluminância elevada para aumentar o reconhecimento das faces das pessoas.

Para a primeira simulação foi utilizada a lâmpada de Descarga convencional modelo HQL 400 W E40 do fabricante Osram. O layout utilizado para a simulação com as lâmpadas HQI para atender a NBR 8995/2013 foi o apresentado pela figura 8, contendo 21 postes de 15 metros de altura com um conjunto de 3 luminárias modelo SPP368 1xSON-TPP400W do fabricante PHILIPS. 
Figura 9 - Layout de Luminárias com Lâmpadas HQI - Estacionamento Descoberto

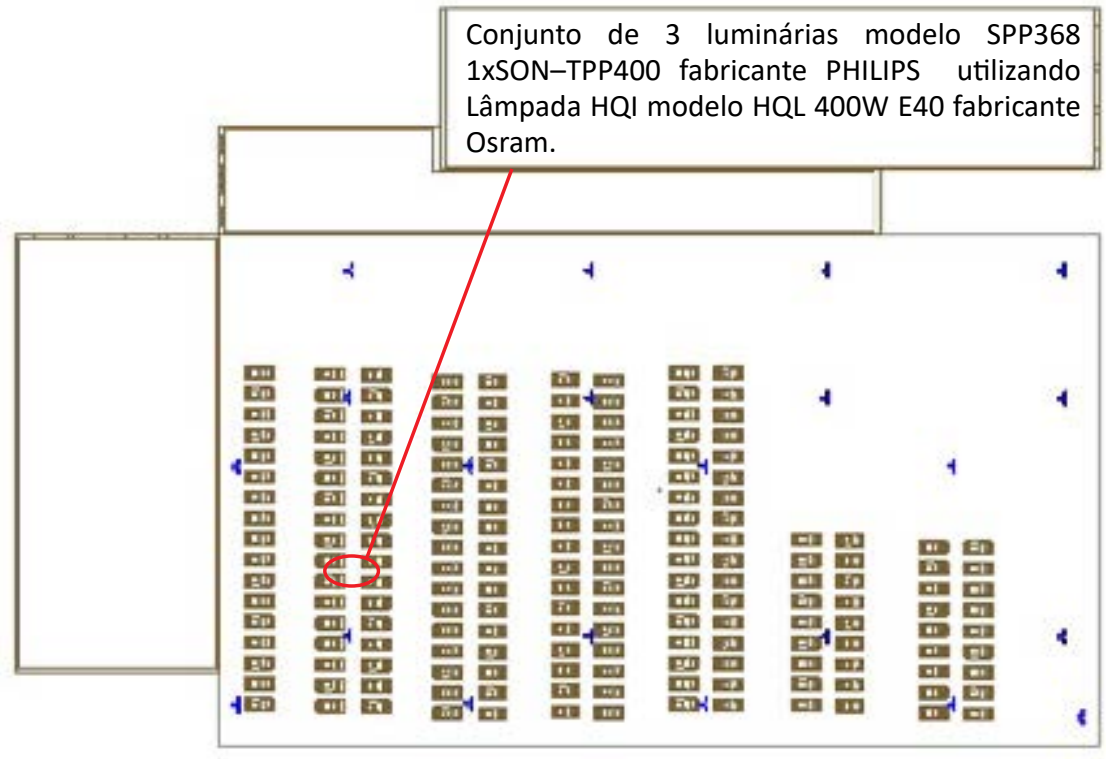

Fonte: (Autor, 2017)

De acordo com o layout apresentado, foram obtidos os resultados de iluminância mantida () mostrados na Tabela 4.

Tabela 1 - Resultado Iluminância Mantida () - Estacionamento Descoberto

\begin{tabular}{|c|c|c|c|c|}
\hline Em (Lux) & Emin (Lux) & Emax (Lux) & Emin / Em & Emin / Emax \\
\hline 69 & 20 & 118 & 0,298 & 0,173 \\
\hline
\end{tabular}

De acordo com os resultados obtidos, verifica-se que o valor de iluminância mantida () se encontra 8\% abaixo do recomendado pela NBR 8995/2013 que determina um valor de iluminância mantida () de 75 Lux. O valor obtido pode ser considerado inicialmente aceitável pois a redução é inferior a 10\% do requerido pela NBR 8995/2013. Com a degradação da lâmpada HQI e queda do seu fluxo luminoso (Im) o valor de iluminância mantida () tende a diminuir apresentando pontos escuros e dificuldade de identificação das fases das pessoas tornando o ambiente menos seguro.

Para a segunda simulação mantendo a mesmo layout de luminárias (figura 9) substituindo as luminárias convencionais por uma luminária de LED, foi utilizada a Luminária 
de LED modelo BGP323 T35 1xECO257-3S/657 DC do fabricante Philips.

Utilizando a Luminária de LED, foram obtidos os resultados de iluminância mantida () mostrados na Tabela 5.

Tabela 5 - Resultado Iluminância Mantida () Luminária de LED - Estacionamento Descoberto

\begin{tabular}{|c|c|c|c|c|}
\hline$E m($ Lux $)$ & $E \min ($ Lux $)$ & $E \max ($ Lux $)$ & $E \min / \mathrm{Em}$ & $\mathrm{Emin} / \mathrm{Emax}$ \\
\hline 98 & 29 & 185 & 0,298 & 0,157 \\
\hline
\end{tabular}

Fonte: (Autor, 2017)

Para a terceira simulação, foi realizado um novo layout de luminárias (figura 10) reduzindo os pontos de luz no ambiente proposto e utilizando a mesma Luminária de LED modelo BGP323 T35 1xECO257-3S/657 DC do fabricante Philips.

Figura 10 - Novo Layout de Luminárias de LED com menos pontos - Estacionamento Descoberto

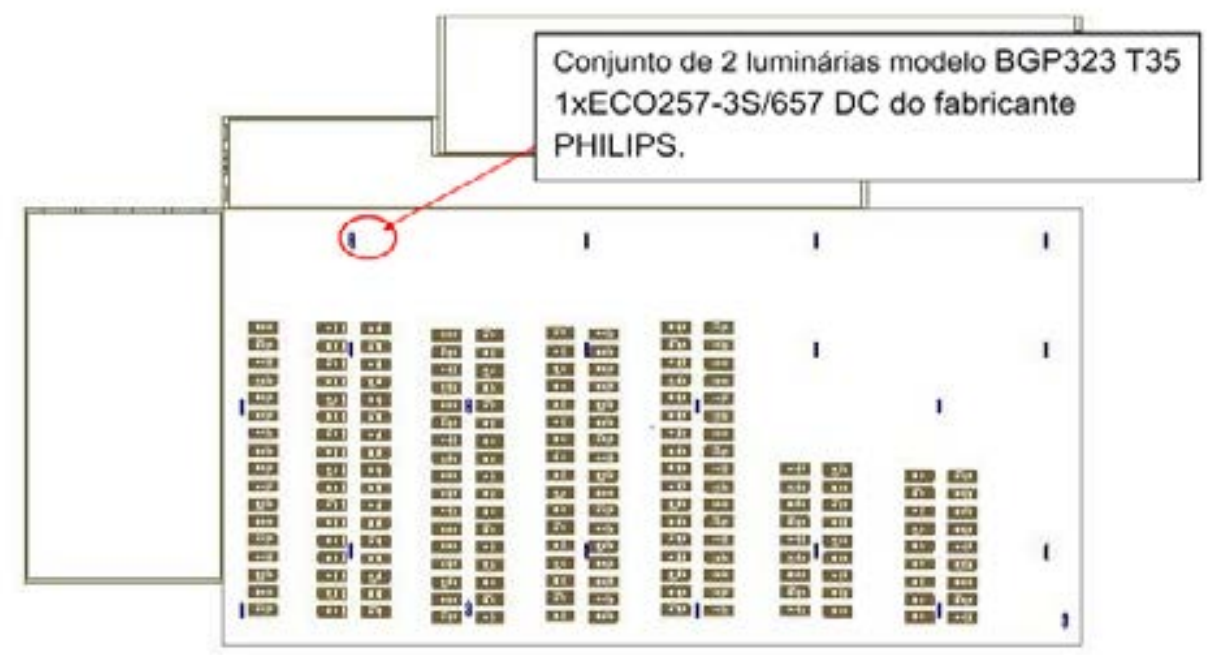

Fonte: (Autor, 2017)

Para o novo layout proposto utilizando a Luminária de LED modelo BGP323 T35 1xECO257-3S/657 DC do fabricante PHILIPS, foram obtidos os seguintes resultados de 
iluminância mantida ().

Tabela 6 - Resultado lluminância Mantida () Luminária de LED menos pontos - Estacionamento Descoberto

\begin{tabular}{|c|c|c|c|c|}
\hline$E m($ Lux) & $E m i n(L u x)$ & $E m a x(L u x)$ & $E \min / E m$ & $E m i n / E m a x$ \\
\hline 66 & 17 & 127 & 0,259 & 0,134 \\
\hline
\end{tabular}

Fonte: (Autor, 2017)

Verifica-se que após a redução dos pontos de luz no ambiente proposto de Estacionamento Descoberto utilizando Luminárias de LED (217 W), a iluminância mantida () retornou próximo aos valores iniciais utilizando lâmpadas de descargas convencionais (HQI) (400 W). Tal resultado atende a NBR 8995/2013, porém o ambiente pode não oferecer uma iluminação segura para as atividades a serem realizadas.

Figura 11 - Comparativos Resultados - Ambiente Externo Estacionamento - Bangu Shopping

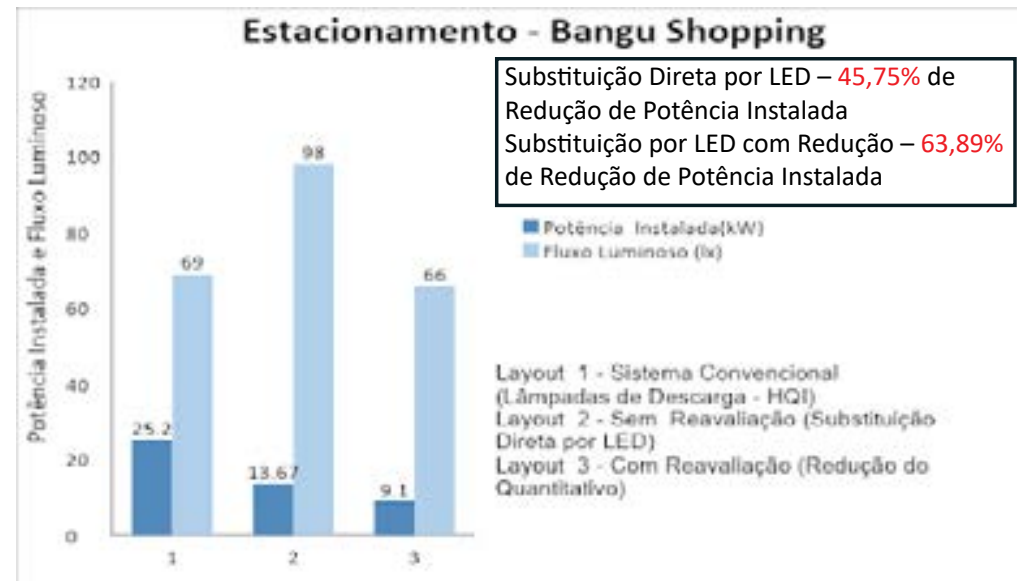

Fonte: (Autor, 2017)

Com a simples substituição foi possível realizar a redução de potência instalada inicialmente de $25200 \mathrm{~W}$ para $13671 \mathrm{~W}$, isto é, uma redução de potência instalada de $45,75 \%$ do valor inicial e ainda assim melhorando a iluminância mantida () inicial de 69 Lux para 98 Lux, o que representa um aumento de aproximadamente $42 \%$, tornando também o ambiente mais seguro para o tipo de atividade desenvolvida no local, confor- 
me apresentado na figura 11.

\section{CONSIDERAÇÕES FINAIS}

Este trabalho tem como objetivo apresentar a diferença entre as lâmpadas fluorescentes tubulares e compactas convencionais perante as novas lâmpadas LED atualmente crescendo no mercado nacional e mundial com o intuito de substituir gradativamente as lâmpadas fluorescentes convencionais. O trabalho apresentado se baseia na conscientização de Engenheiros e Técnicos que atuem no ramo de iluminação para que não ocorram dois fatos importantes na substituição das lâmpadas fluorescentes convencionais por lâmpadas de LED, conforme apresentado; a simples substituição das lâmpadas que pode ocasionar um excesso de intensidade de luz dentro dos ambientes, e um consumo desnecessário de energia elétrica para o ambiente onde ocorreu a substituição.

Os excessos de luminosidade dentro dos ambientes, além de estarem superdimensionados em referência às normas NBR 5413:1992, NBR 8995:2013 e NBR 5101:2012, podem ocasionar desconforto, ofuscamento, ressecamento de retina, entre outros problemas visuais para os usuários ali presentes.

Outro ponto importante referente ao excesso de luminosidade dentro dos ambientes é a questão do consumo excessivo de energia elétrica, tendo em vista que o intuito das substituições é em sua grande maioria a redução do consumo referente aos sistemas de iluminação instalados. Os sistemas de iluminação chegam a representar em média $20 \%$ do valor final do consumo de energia elétrica nas contas de energia.

Foi possível realizar a redução dos quantitativos de Luminárias apresentando assim uma eficiência luminosa dentro dos padrões requeridos pelas normas NBR 5413:1992, NBR 8995:2013 e NBR 5101:2012 e evitar um consumo excessivo de energia elétrica para os ambientes, o que impacta diretamente no valor final de conta de energia elétrica.

Ambientes como Salas de Aula, onde foi possível realizar uma redução de luminárias mantendo uma iluminância mantida () dentro do solicitado pela NBR 8995:2013, apresentaram uma redução de potência instalada inicial de $720 \mathrm{~W}$ para um valor final de 396 W, isto é, em torno de $45 \%$ de redução de potência instalada, o que pode ser 
extrapolado para uma escola ou universidade onde mais de $90 \%$ das instalações é predominantemente salas de aula, ou seja, de acordo com o estudo realizado seria possível efetuar uma redução próxima de $45 \%$ do total de carga instalada de iluminação.

Como apresentado nos resultados das simulações, figura 11, o Bangu Shopping ao realizar a substituição da iluminação convencional do seu estacionamento para um sistema de iluminação LED, além de obter a redução de consumo de energia elétrica total do estacionamento em aproximadamente $42 \%$, pode permanecer com o mesmo quantitativo de pontos de luz, pois, ainda que reduzindo o consumo foi possível melhorar a iluminância mantida () próxima dos 100 lux, nos pátios de estacionamento, aumentando a sensação de segurança para os usuários e acabando com pontos escuros existentes anteriormente.

O mesmo exemplo do Shopping Bangu pode ser seguido por outros Shoppings Centers que ainda não realizaram tal investimento em sistemas de iluminação, visando os benefícios vão além da simples redução de carga instalada.

\section{REFERÊNCIAS}

ASSOCIAÇÃO BRASILEIRA DE NORMAS TÉCNICAS. NBR-5413: Norma Brasileira de Iluminação de Interiores. 1992

ASSOCIAÇÃO BRASILEIRA DE NORMAS TÉCNICAS. NBR-5101: Norma Brasileira de Iluminação Pública - Procedimentos. 2012

ASSOCIAÇÃO BRASILEIRA DE NORMAS TÉCNICAS. NBR-8995: Norma Brasileira de Iluminação de Ambientes de Trabalho. 2013. 
EMPALUX, Informações Luminotécnicas. Disponível em: < http://www.empalux.com. br/?a1=l> Acesso em: 23 maio 2017.

ELEUTÉRIO, Rafael Vidal. Absorção de Luz. InfoEscola. 2012, Disponível em: < http:// www.infoescola.com/fisica/absorcao-de-luz> Acesso em 12 maio 2017.

FAU - Faculdade de Arquitetura e Urbanismo da Universidade de São Paulo, Conceito Fundamentais Grandezas Luminosas, Disponível em:

<http://www.fau.usp.br/arquivos/disciplinas/au/aut0213/Material_de_Apoio/03_-_ la._Conceito_Fundamentais_(grandezas_Luminosas).pdf>Acesso em: 27 maio 2017.

FERREIRA, Juliana Zandona. Estudo Comparativo Entre Lâmpadas Fluorescentes Tubulares T8 e Tubulares de LED. Juliana Zandona Ferreira - Curitiba - PR, UTFPR. 2014.

IORJ - Instituto de Oftalmologia do Rio de Janeiro.OLHO SECO. Disponível em:

<http://www.iorj.med.br/olho-seco/> Acesso em 12 maio 2017.

LIMA, Valquíria Aparecida Alcantara. Estudo Comparativo Entre Lâmpada com LED de Alta Potência e Lâmpadas Comuns, Considerando a Viabilidade Econômica. Valquíria Aparecida Alcantara Lima. - Curitiba - PR, UTFPR. 2013.

LUMICENTER, Informações Técnicas, Disponível em:<http://www.lumicenteriluminacao.com.br/arquivos/info_tecnicas_lumicenter.pdf $>$

Acesso em: 24 maio 2017.

OSRAM, Manual Luminotécnico Prático. São Paulo, 2007. Disponível em: <http://www. iar.unicamp.br/lab/luz/ld/Livros/ManualOsram.pdf> Acesso em: 24 maio 2017. 
PADILHA, M.; JUNG, F.; RODRIGUES, E. ESTUDO COMPARATIVO ENTRE LÂMPADAS FLUORESCENTES E LED APLICADO NO IFC - CAMPUS LUZERNA. VIII MICTI - Mostra Nacional de Iniciação Cientifica e Tecnológica Interdisciplinar, Luzerna - SC, 2015.

PORTAL BRASIL. Lâmpadas incandescentes saem do mercado a partir de julho. Disponivel em: <http://www.brasil.gov.br/infraestrutura/2016/06/lampadas-incandecentes-saem-do-mercado-a-partir-de-julho> Acesso em 12 maio 2017.

SALVETTI, Alfredo Roque. A História da Luz. Editora Universidade Federal Mato Grosso do Sul - UFMS, Campo Grande, 2008. 